Real-time Probabilistic Nowcasts of UK Quarterly GDP Growth using a Mixed-Frequency Bottom-up Approach

Ana Beatriz Galvão and Marta Lopresto

ESCoE Discussion Paper 2020-06

May 2020 
Real-time Probabilistic Nowcasts of UK Quarterly GDP Growth using a Mixed-Frequency Bottom-up Approach

Ana Beatriz Galvão and Marta Lopresto

ESCoE Discussion Paper No. 2020-06

May 2020

\begin{abstract}
We propose a nowcasting system to obtain real-time predictive intervals for the firstrelease of UK quarterly GDP growth that can be implemented in a menu-driven econometric software. We design a bottom-up approach: forecasts for GDP components (from the output and the expenditure approaches) are inputs into the computation of probabilistic forecasts for GDP growth. For each GDP component considered, mixed-datasampling regressions are applied to extract predictive content from monthly and quarterly indicators. We find that predictions from the nowcasting system are accurate, in particular when nowcasts are computed using monthly indicators 30 days before the GDP release. The system is also able to provide well-calibrated predictive intervals.
\end{abstract}

Keywords: nowcasting, GDP growth, mixed frequency regression, forecast combination, probabilistic forecasts

JEL classification: C53, E32

Ana Beatriz Galvão, University of Warwick, ana.galvao@wbs.ac.uk and Marta Lopresto, Bank of England, marta.lopresto@bankofengland.co.uk.

Published by:

Economic Statistics Centre of Excellence

National Institute of Economic and Social Research

2 Dean Trench St

London SW1P 3HE

United Kingdom

www.escoe.ac.uk

ESCoE Discussion Papers describe research in progress by the author(s) and are published to elicit comments and to further debate. Any views expressed are solely those of the author(s) and so cannot be taken to represent those of the Economic Statistics Centre of Excellence (ESCoE), its partner institutions or the Office for National Statistics (ONS). 


\title{
Real-time Probabilistic Nowcasts of UK Quarterly GDP Growth using a Mixed-Frequency Bottom-up Approach ${ }^{\dagger}$
}

\author{
Ana Beatriz Galvão, University of Warwick ${ }^{\ddagger}$ \\ Marta Lopresto, Bank of England
}

May, 2020

\begin{abstract}
We propose a nowcasting system to obtain real-time predictive intervals for the first-release of UK quarterly GDP growth that can be implemented in a menu-driven econometric software. We design a bottom-up approach: forecasts for GDP components (from the output and the expenditure approaches) are inputs into the computation of probabilistic forecasts for GDP growth. For each GDP component considered, mixed-data-sampling regressions are applied to extract predictive content from monthly and quarterly indicators. We find that predictions from the nowcasting system are accurate, in particular when nowcasts are computed using monthly indicators 30 days before the GDP release. The system is also able to provide well-calibrated predictive intervals.
\end{abstract}

Keywords: nowcasting, GDP growth, mixed frequency regression, forecast combination, probabilistic forecasts

JEL codes: C53, E32

\footnotetext{
${ }^{+}$This research has been funded by the ONS as part of the research programme of the Economic Statistics Centre of Excellence (ESCOE), and mainly carried out during the time that Marta Lopresto was working the National Institute of Economic and Social Research (NIESR). We would like to thank Garry Young and Amit Kara for their suggestions and support. Any views expressed are solely those of the author(s) and so cannot be taken to represent those of the Bank of England or to state Bank of England policy. This paper should therefore not be reported as representing the views of the Bank of England or members of the Monetary Policy Committee, Financial Policy Committee or Prudential Regulation Committee.

‡ Corresponding author: Prof. Ana Galvao, Warwick Business School, University of Warwick, CV4 7AL Coventry; ana.galvao@wbs.ac.uk
} 


\section{Introduction}

Measurements of economic activity are vital for tracking the economy and affect the decision making of market participants, businesses and policymakers. Nowcasting models, surveyed by Banbura et al. (2013), are designed to accommodate the fact that economic and financial variables are typically available at monthly and quarterly frequencies and may be subject to publication delays. Trackers of the UK economy have recently (July 2018) to deal with a change in the Office of National Statistics (ONS) release calendar for quarterly real GDP, a key UK economic activity measure. Two significant changes were implemented. First, quarterly GDP is now released with a 40-day delay instead of a 25-day delay. Second, a measure of monthly GDP is published every month with a 40-day delay to the reference month. The increase delay in the publication of the first quarterly GDP release implies that the ONS can now employ data from the expenditure approach in addition to the output approach to compute the first quarterly GDP estimate. ${ }^{1}$ The new ONS release calendar provides more information for trackers of the UK economy because of the publication of monthly GDP, but with the caveat that the first announcement of quarterly GDP, the main target for policy-making and measurement of current economic conditions, is postponed.

The reported changes in the publication calendar for such key quarterly economic variable motivates our proposal of a new mixed-frequency nowcasting system for the UK quarterly GDP growth rate able to exploit the predictive content of monthly economic variables. We aim to provide accurate predictions of quarterly GDP growth computed one, two and three months ahead of the ONS first release. In addition to point predictions, as it is usually the output of nowcasting statistical models (Banbura et al., 2013), we also aim to provide predictive intervals. The advantage of computing prediction intervals is that we can communicate the uncertainty around the nowcasts. As forecasting uncertainty may change over time due to changes in the degree of predictability of the first UK GDP release, our nowcasts and predictive intervals provide additional information to policy makers and

\footnotetext{
${ }^{1}$ The ONS calculates GDP using the output (Gross Value Added), the expendidure and income approaches. For each case, the GDP is a sum of a different set of components. The ONS publishes a unique UK GDP value and discrepancies among the three approaches are eliminated as part of the revision process using a triangulation scheme.
} 
economic agents that make decisions based on the current state of the economy. Predictive intervals offer a range of possible values at a given probability and allow the computation of the probability of key events such as the probability of negative growth.

In this paper, we propose to measure forecasting uncertainty using the past nowcasting accuracy of predictions obtained using a bottom-up approach. Our bottom-up approach combines nowcasts computed for a set of GDP components, such as, the indices of production and services, and aggregate consumption and investment. The method, as in the case of the GDPnow (Higgins, 2014), published regularly by the Atlanta Fed, is inspired by the idea of building a nowcast model by replicating the way statistical offices compute GDP estimates. The GDPnow approach makes use of "bridge equations" that link the quarterly indicators to monthly indicators. The approach requires the computation of forecasts for the monthly components before a forecast for the quarterly indicator is computed using a quarterly-databased regression. The empirical results in Clements and Galvao (2008) suggest that using Mixed Data Sampling (MIDAS) regression which exploits the information contained within the monthly series achieves more accurate forecasts than using the "bridge equation" approach.

This paper proposes a bottom-up mixed-frequency nowcasting system to predict earlier releases of UK GDP growth in real time. We use real-time data from the Bank of England realtime dataset and the ONS real-time downloadable spreadsheets and revision triangles. As suggested by Clements and Galvao (2008), mixed-frequency regressions are an effective way to employ current monthly information directly when nowcasting in real time. An additional advantage of the MIDAS approach is that it is computationally inexpensive in contrast to dynamic factor models in Banbura et al (2013). The MIDAS approach can be easily implemented in standard econometric packages such as Eviews. Furthermore, Anesti et al. (2017) suggest that a combination of MIDAS regressions provides more accurate point forecasts for UK GDP than dynamic factor models.

By building a nowcasting model for UK GDP growth using mixed sampling models, this paper contributes to the literature on the use of MIDAS models for forecasting (Clements and Galvao, 2008; Foroni et al., 2015), and on UK GDP nowcasting models, including earlier contributions by Mitchel et al. (2005) and Mitchel (2009).

Our approach explicitly targets the first release of quarterly UK GDP growth to deliver well-calibrated predictive intervals in real time. Later ONS releases of GDP are based on a more 
extensive information set and are considered to be better estimates of historical GDP growth. The data revisions process of UK GDP growth causes an additional layer of uncertainty to the measurement of economic activity as Galvao and Mitchell (2019) have suggested based on ONS data and Bank of England backcasts. As a consequence, if we target revised GDP growth, we not only have to consider how to forecast the first release of quarterly GDP but also how to predict future data revisions. Because revised GDP has a long publication delay (3 or 4 years), we assume that nowcasters are more interested in tracking earlier releases and are concerned with forecasting uncertainty instead of data uncertainty.

As a consequence, we estimate our forecasting models using only first releases of quarterly GDP growth. Point forecasts for revised GDP growth computed with forecasting models calculated using first estimates should be adjusted if the mean of expected data revisions is nonzero (as suggested by Clements and Galvao, 2013). The alternative approach of estimating the forecasting model using the latest vintage of data available at each point in time is not recommended. Clements (2017) and Clements and Galvao (2018) explain the deficiencies of employing the lastest data to deliver accurate real-time forecasts, particularly if data revisions remove earlier measurement errors.

The remaining of this paper is structured as follows. In section 2, we describe our nowcasting methodology, starting with our method to compute predictive densities using a forecasting combination. Then we present the models employed for computing forecasts for each GDP component considered. Section 3 describes the dataset employed, including data sources and the selection of indicators for each GDP component. Section 4 presents our realtime forecasting results. We start by showing the forecast performance of a combination of GDP components from the expenditure and the output approaches. We evaluate the real-time point forecast and predictive interval accuracy of our nowcasting system in comparison with alternative forecasting methods. Section 5 summarises our methodological and empirical contributions.

\section{A bottom-up Mixed Frequency Nowcasting Methodology}

In this section, we present the three steps of our mixed-frequency bottom-up nowcasting system. We start from the last (top) step, and the presentation of the two intermediary steps are in the subsections that follow. 


\subsection{Computing Predictive Intervals for Real-time UK GDP Growth}

Our target is to predict the first release of quarterly UK GDP growth $y_{t}$ using information up to $t-h$, that is, $y_{t \mid t-h}$. We consider forecasting horizons in months (or sets of 30 days). Because of the current 40-day delay in the GDP publication and the fact that monthly and quarterly indicators may be available earlier, we set $h=0,1,2 . y_{t \mid t}$ is a prediction of the first release of GDP growth using information up to 30 days ahead of ONS release of GDP. The information set considered includes data for some indicators for all months of the current quarter. The forecast $y_{t \mid t-1}$ uses information up to 60 days before GDP is released and only contains data for the first two months of the reference quarter for most monthly indicators. $y_{t \mid t-2}$ uses information up to 90 days before the GDP release and typically only includes data on the first month of the reference quarter.

Because forecasts are computed using estimated models, the point forecast is $\widehat{y_{t \mid t-h}}$. To obtain predictive intervals, we assume a Gaussian predictive density, so we need an estimate of the forecasting uncertainty $\widehat{\sigma_{t \mid t-h}}$. Both the point forecasts and forecasting uncertainties are estimated by using a regression that combines forecasts obtained using GDP components of the output $\widehat{y_{t \mid t-h}^{\text {out }}}$ and the expenditure $\widehat{y_{t \mid t-h}^{\text {exp }}}$ approach. To estimate the combination weights, we split the out-of-sample period $\mathrm{P}$ into $P_{1}$ and $P_{2}$ where $P_{1}+P_{2}=P$. We estimate the combination weights for $t=R+1, \ldots, R+P_{1}$ where $R$ is the last observation of the in-sample period, and the total number of observations is $R+P$. As discussed in detail in section 3 , we divide an out-of-sample period of approximately 10 years into two subperiods of 5 years. The combination weights are computed using the following regression:

$$
y_{t}=\lambda_{h} \widehat{y_{t \mid t-h}^{\text {out }}}+\left(1-\lambda_{h}\right) \widehat{y_{t \mid t-h}^{\text {exp }}}+\xi_{t} .
$$

The advantage of using equation (1) is that it is equivalent to the auxiliary regression of forecasting encompassing tests. The implication is that forecasts from each GDP-measurement approach are weighted based on their past performance in predicting the first release of GDP. We can also evaluate the statistical significance of $\lambda$ to verify the usefulness of our bottom-up approach, that is, whether components from both GDP approaches contribute to the GDP growth nowcasts.

There is a second advantage of using (1) to combine forecasts, $\operatorname{var}\left(\xi_{t}\right)$ measures the remaining uncertainty when using the forecasts for predicting quarterly GDP growth. This 
implies that $\widehat{\sigma_{t \mid t-h}}=\sqrt{\operatorname{var}\left(\hat{\xi}_{t}\right)}$. Then 50\%, 75\% and 90\% nowcast predictive intervals are obtained by assuming Gaussian predictive densities and by computing a consistent estimate of $\operatorname{var}\left(\xi_{t}\right)$. We are, then, able to assess the uncertainty around a central nowcast, which is an important feature for decision making when tracking the UK economy since it provides information on the likelihood of the central path. Nowcasting models usually overlook the computation of an estimate of forecasting uncertainty.

By using $\operatorname{var}\left(\xi_{t}\right)$, we are disregarding parameter uncertainty. When predicting GDP growth, forecasting uncertainty is usually large in comparison with parameter and modelling uncertainty. In section 4, we check whether the predictive intervals obtained from this approach deliver well-calibrated intervals. In case of correct calibration, we have an empirical validation of our nowcasting system specification.

We evaluate our nowcasting system by using $\hat{\lambda}_{h}$ and $\widehat{\sigma_{t \mid t-h}}$ estimated by least squares using (1) and observations up to $P_{1}$ to compute real-time nowcasts for the second subperiod $=R+P_{1}+1, \ldots, R+P$. The point forecasts are $\widehat{y_{t \mid t-h}}=\hat{\lambda}_{h} \widehat{y_{t \mid t-h}^{\text {out }}}+\left(1-\hat{\lambda}_{h}\right) \widehat{y_{t \mid t-h}} \overline{\text { exp }}$ and the predicted standard error is $\widehat{\sigma_{t \mid t-h}} \cdot(1-\alpha) \%$ predictive intervals are computed as:

$$
\left[\widehat{y_{t \mid t-h}}-Z^{\alpha / 2} \widehat{\sigma_{t \mid t-h}}, \widehat{y_{t \mid t-h}}+Z^{1-\alpha / 2} \widehat{\sigma_{t \mid t-h}}\right]
$$

where $Z^{\alpha / 2}$ and $Z^{1-\alpha / 2}$ are the required quantiles of the standard normal distribution.

In section 4, we estimate the combination weights in regression (1) with either a rolling window of size $P_{1}$ over the second out-of-sample period or with an increasing number of observations.

\subsubsection{Time-varying forecasting uncertainty}

Empirically, the period $\mathrm{P}_{1}$ of the out-of-sample period covers the quarters from 2008Q1 up to 2012Q4, while $P_{2}$ covers the quarters from 2013Q1 up to 2018Q3. UK GDP growth over the second period exhibits lower variability than in the first period. As a consequence, predictive intervals computed as described earlier - assuming that forecasting uncertainty does not change over the period - have some difficulties in delivering well-calibrated predictions of uncertainty. This is the case even if we use rolling windows to estimate the weights and to compute the predicted variance using equation (1). 
Therefore, in an attempt to improve the calibration of the predictive intervals, we estimate a $\mathrm{GARCH}(1,1)$ process for the conditional variance, assuming the following:

$$
\operatorname{var}\left(\xi_{t}\right)=h_{t}=\varpi_{0}+\varpi_{1} \xi_{t-1}+\varpi_{1} h_{t-1}
$$

The $\operatorname{GARCH}(1,1)$ is estimated with an increasing sample window over the second part of the out-of-sample period. We compute point forecasts as before, and the predicted variance $\widehat{\sigma_{t \mid t-h}}=\hat{h}_{t}$ is the value for the last observation in the estimation window.

\subsection{Computing point forecasts for expenditure and output components}

As described earlier, the top layer of our three-step nowcasting system combines forecasts from two different statistical approaches for the computation of GDP. In this subsection, we explain how we obtain the forecasts for each GDP component.

We consider at least three components from the output and the expenditure approaches applied in the computation of the UK GDP. In the case of the expenditure approach, we compute forecasts for quarterly consumption growth $C_{t}$, quarterly investment growth $I_{t}$, quarterly growth of exports $X_{t}$, and imports $M_{t}$. For the output approach, we compute forecasts for quarterly growth rates of the index of services $I O S_{t}$, the index of production $I O P_{t}$ and the index of construction $I O C_{t}$. We explain how we calculate the forecasts for each one of these components for the real-time out-of-sample period in the next subsection.

The weights for the expenditure approach are estimated using the following regression:

$$
y_{t}=\gamma_{1} C_{t}+\gamma_{2} I_{t}+\gamma_{3} X_{t}+\gamma_{4} M_{t}+\epsilon_{t}
$$

and the ones for the output approach use:

$$
y_{t}=\delta_{1} I O S_{t}+\delta_{2} I O P_{t}+\delta_{3} I O C_{t}+\epsilon_{t}
$$

The regressions are estimated using either a rolling window of 20 quarters or increasing windows of data during the out-of-sample period. We re-scale the weights such that they sum up to one. In practice, the weights are stable over time, and setting them to their average values usually does not affect forecasting performance. To note, we do not give any causal interpretation to these weights.

To compute forecasts for aggregate output, we employ these weights to combine forecasts for components of each approach. Forecasts for GDP using the output-approach are:

$$
\widehat{y_{t \mid t-h}^{\text {out }}}=\hat{\delta}_{1} I \widehat{O S_{t \mid t}-h}+\hat{\delta}_{2} I \widehat{O P_{t \mid t}-h}+\hat{\delta}_{3} I \widehat{O C_{t \mid t}-h}
$$


and for the expenditure-approach:

$$
\widehat{y_{t \mid t-h}^{e x p}}=\hat{\gamma}_{1} \widehat{C_{t \mid t-h}}+\hat{\gamma}_{1} \widehat{I_{t \mid t-h}}+\hat{\gamma}_{3} \widehat{X_{t \mid t-h}}+\hat{\gamma}_{4} \widehat{M_{t \mid t-h}} .
$$

We compute forecasts for $t=R+P_{1}+1, \ldots, R+P$ using forecasts for each component based on MIDAS models as described in section 2.3. Note that we use only past data to estimate weights, so to have a genuinely out-of-sample exercise.

\subsection{Employing MIDAS models to predict GDP components}

Clements and Galvao (2008) show how mixed data sampling models are useful for forecasting US GDP growth when using monthly indicators. In this section, we describe how we employ MIDAS regressions to predict each one of the six GDP components listed in the previous section.

Suppose that our target variable, the growth rate of a GDP component, is $x_{t}$, which is only available quarterly, but we have a set of predictors $z_{1 t}, \ldots, z_{k t}$ which are available monthly. The MIDAS regression is:

$$
x_{t}=\alpha_{0}+\sum_{i=1}^{p} \alpha_{i} x_{t-i}+\sum_{i=1}^{k} \beta_{i, h} \sum_{j=1}^{m} w\left(\theta_{i, h}\right) z_{i, t-h-j}+\varepsilon_{t} .
$$

where $\sum_{i=1}^{p} \alpha_{i} x_{t-i}$ are quarterly lags of the dependent variable and $m$ is the number of monthly lags for each predictor. Note that as before the forecasting horizon $h$ is measured in months and we are implicitly assuming that we do not consider forecast horizons longer than a quarter as $h=0,1,2$. The coefficients of each lagged monthly predictor are defined by the slope $\beta_{i, h}$ multiplied by the weights for each lag that follow a weight function with parameters $\theta_{i, h}$.

In particular, we use the Polynomial Distributed Lag / Almon Lag model as weighting function, that uses an $m$ degree polynomial to weight the lags of the monthly regressors to predict the quarterly variable. As explained in Pettenuzo et al (2016), the advantage of using the Almon Lag polynomial weighting function is that equation (4) can be rewritten to exploit the fact that the slope of each regressor $\beta_{i, h}$ can be computed by using the usual least square formula, once the adequate powers of the regressors for each monthly lag are obtained. Almon weighting functions are then used to avoid the numerical optimization issues when applying alternative weighting function such as for the exponential Almon lags function implemented in Clements and Galvao (2008). 
For two components of the output approach, such as the index of services and the index of production, monthly data are also available. As a consequence, we estimate the MIDAS regression for these components with monthly autoregressive lags, included in the same way as the monthly regressors $z_{i t}$.

The MIDAS regressions, as in eq. (4), are estimated for each component separately. We also estimate one model for each horizon $h=0,1,2$ using an increasing sample window over the out-of-sample period. Point forecasts are obtained by direct forecasting. As anticipated in the introduction, because the GDP components are subject to data revisions, we use a time series of first releases for each quarterly growth rate series. The above choices imply we compute real-time forecasts using only information up to $t$ at each forecast origin during the out-of-sample period. These real-time forecasts, $I \widehat{I S S_{t \mid t} h}, I O P_{t \mid t-h}, I O C_{t \mid t-h}, \widehat{C_{t \mid t-h}}, \sqrt{t \mid t-h}$, $\widehat{X_{t \mid t-h}}, \widehat{M_{t \mid t-h}}$, are then inputs for the next steps described earlier.

\section{The Real-time dataset}

We start by describing the real-time dataset employed. Table 1 lists each GDP component used and the source of real-time data vintages.

We set the period between 2008Q1 and 2018Q3 as our out-of-sample period, and observations from 1997Q1 are employed to estimate forecasting models and weights. We use the real-time vintages for each one of the seven GDP components considered, as described in Table 1, to compute a time series of first releases in growth rates. For some GDP components, as indicated in Table 1, for most the GDP components, real-time data is available from 1997. In some cases, when only available from 2006, we use values from the first available vintage for the earlier history. Note, however, that the vintage data covers all the out-of-sample period, so our exercise is indeed a real-time exercise.

Our approach to deal with data revisions when forecasting GDP components removes the impact of data revisions of all GDP components when estimating the forecasting models. This approach improves the accuracy of forecasting models targeting early releases of UK GDP growth as the analytical results in Clements and Galvao (2013) indicate. In contrast to Clements and Galvao (2013), we do not use real-time vintages for the right-hand side of the regression as our forecasting models are estimated using only the time series of first releases. As a consequence, we ignore data revisions on longer lags of the predictors that could improve the 
accuracy of the prediction. In practice, the impact of this modelling choice is likely to be small. At the same time, it has the advantage of allowing us to update and estimate our real-time nowcasting system using a standard package software as Eviews.

Table 1: Data description - GDP Components

\begin{tabular}{|c|c|c|c|c|}
\hline & Definition & Source & $\begin{array}{l}\text { Vintages } \\
\text { Available }\end{array}$ & $\begin{array}{l}\text { ONS } \\
\text { code }\end{array}$ \\
\hline $\mathrm{Y}$ & GDP at market prices & ONS & 1997Q1-2018Q3 & $A B M I$ \\
\hline $\mathrm{C}$ & Household Consumption & $\begin{array}{l}\text { BofE } \\
\text { ONS }\end{array}$ & $\begin{array}{l}\text { 1998Q3 - 2016Q2 } \\
\text { 2016Q3-2018Q3 }\end{array}$ & $A B J R$ \\
\hline I & $\begin{array}{l}\text { Gross Fixed Capital } \\
\text { Formation }\end{array}$ & $\begin{array}{l}\text { BofE } \\
\text { ONS }\end{array}$ & $\begin{array}{l}\text { 1998Q3 - 2016Q2 } \\
\text { 2016Q3-2018Q3 }\end{array}$ & NPQT \\
\hline$x$ & $\begin{array}{l}\text { Export (total: goods and } \\
\text { services) }\end{array}$ & $\begin{array}{l}\text { BofE } \\
\text { ONS }\end{array}$ & $\begin{array}{l}\text { 1997Q1 - 2016Q2 } \\
\text { 2016Q3-2018Q3 }\end{array}$ & IKBK \\
\hline M & $\begin{array}{l}\text { Import (total: goods and } \\
\text { services) }\end{array}$ & $\begin{array}{l}\text { BofE } \\
\text { ONS }\end{array}$ & $\begin{array}{l}\text { 1997Q1 - 2016Q2 } \\
\text { 2016Q3-2018Q3 }\end{array}$ & IKBL \\
\hline IOS Q & Index of Services, Monthly & $\begin{array}{l}\text { BofE } \\
\text { ONS }\end{array}$ & $\begin{array}{l}\text { 1997Q1 - 2016Q2 } \\
\text { 2016Q3-2018Q3 }\end{array}$ & L2NC \\
\hline IOS M & Index of Services, Monthly & $\begin{array}{l}\text { Anesti et al (2018) } \\
\text { ONS }\end{array}$ & $\begin{array}{l}\text { 2006M07- } \\
2016 \mathrm{M} 12 \\
2017 \mathrm{M} 01- \\
2018 \mathrm{M} 11\end{array}$ & \\
\hline IOP Q & $\begin{array}{l}\text { Index of Production, } \\
\text { Quarterly }\end{array}$ & $\begin{array}{l}\text { Anesti et al (2018) } \\
\text { BofE } \\
\text { ONS }\end{array}$ & $\begin{array}{l}\text { 2006Q3-2011Q1 } \\
\text { 2011Q2 - 2016Q2 } \\
\text { 2016Q3-2018Q3 }\end{array}$ & L2KQ \\
\hline IOP M & $\begin{array}{l}\text { Index of Production, } \\
\text { Monthly }\end{array}$ & Anesti et al. (2018) & $\begin{array}{l}\text { 2006M07- } \\
\text { 2012M12 }\end{array}$ & \\
\hline IOC Q & Index of Construction & $\begin{array}{l}\text { BofE } \\
\text { ONS }\end{array}$ & $\begin{array}{l}\text { 1997Q1 - 2016Q2 } \\
\text { 2016Q3-2018Q3 }\end{array}$ & L2N8 \\
\hline
\end{tabular}

Note: The forecasting models are estimated using observations from 1997Q1. When real-time data is only available later, we use observations from the first available vintage (2006Q3 for IOP, for example).

We consider eleven monthly indicators as predictors in the MIDAS regressions described in equation (4). Table 2 lists all predictors considered and the data source. These indicators relate to both statistical-office data, such as retail sales, and survey-based data. The latter are indicators of business activity, consumer confidence, and production expectations, 
among others. Survey-based indicators are timelier than official data so that they can provide useful early signals.

We indicate in Table 2 the models where the predictors are employed. On average, we use three predictors $(k=3)$ for each GDP component.

Table 2: Data Description - Monthly Predictors

\begin{tabular}{l|l|l}
\hline Regressor & Source & Predictor for: \\
\hline Retail sales & ONS & C \\
Consumer Confidence & DGECFIN & I, M \\
Consumer Confidence & GFK-Markit & C, IOP \\
Business Climate & DGECFIN & I, M \\
CBI Retail Orders & CBI & I, X, M \\
CBI Motor Trades & CBI & IOS \\
Export Order & DG ECFIN & X \\
Sterling Effective Exchange Rate & BofE & X \\
Investment Intentions & BofE & IOP \\
Construction Output & BofE & IOC \\
\hline
\end{tabular}

\section{An Empirical Forecasting Exercise}

In this section, we address the following empirical research questions. Is it worth using a bottom-up approach to predict UK GDP growth? The second question is: are the nowcasting predictive intervals well-calibrated? The final point of enquiry is an assessment of the relative point forecast accuracy performance of the proposed mixed-frequency nowcasting system in comparison with professional forecasters such as the Bank of England and NIESR.

\subsection{Evaluating the Predictive Content of the Expenditure and the Output Approaches}

An advantage of our modelling approach is that we can compare the accuracy of forecasts obtained using output and expenditure GDP components. The computation of forecasts $\widehat{y_{t \mid t-h}^{\text {out }}}$ and $\widehat{y_{t \mid t-h}^{\text {exp }}}$ requires the estimation of the combination weights as described earlier. Table 3 provides estimates of the weights calculated using regressions (2) and (3) estimated using actual data of first releases of GDP and its components on both the expenditure and output sides. Two types of regressions are run for each equation: (i) an increasing sample regression estimated over the period 1997Q1-2018Q3, and (ii) a rolling sample regression of 20 
quarters estimated over the period 2003Q1-2018Q3. Note that Table 3 reports the average weight for the period 2007Q4-2018Q3, while time-varying weights are the ones used to combine forecasts. These weights are then re-scaled such that their sum is equal to 1 .

Table 3: Estimated weights for components on expenditure and output sides

\begin{tabular}{llcccc}
\hline Expenditure side - equation (2) & & C & I & X & M \\
\hline & Increasing sample & 0.860 & 0.102 & 0.113 & -0.075 \\
& Rolling sample & 0.867 & 0.101 & 0.108 & -0.076 \\
\hline & & & & & \\
\hline Output side - equation (3) & & IOS & IOP & IOC & \\
\hline & Increasing sample & 0.796 & 0.166 & 0.038 & \\
& Rolling sample & 0.819 & 0.121 & 0.059 & \\
\hline
\end{tabular}

Notes: The weights reported are the average of the coefficients estimated for each window for the period 2007Q4-2018Q3 rescaled to sum to 1 . The rolling window is of 20 quarters. The increasing window uses data from 1997Q1. We use a time series of first releases for each variable to estimate weights.

As expected, based on the GDP composition, the most considerable influence is given to consumption (86\%) in the expenditure approach and the index of services (80\%) in the output approach. The smallest weights are applied to imports in the case of the expenditure approach (-7\%) and the index of construction (5\%) in the case of the output approach. An inspection of the weights over time suggests that they are stable. As expected, weights computed with rolling windows vary relatively more over time, but the differences are small.

After computing the weights of the components of GDP from the expenditure and output approaches, MIDAS regressions for each variable, as equation (4), are estimated with data from 1997Q1 to compute real-time out-of-sample forecasts for the period 2008Q12018Q3. At each new forecast origin from 2007Q4 onwards, we add one more quarter of information and re-estimate the MIDAS regressions coefficients. These regressions use the same set of monthly explanatory variables described in Table 2, while the number of lags for each variable may change since we use information criteria to automate the lag selection. The forecasts generated for the components are then aggregated using the $\{t-1\}$ weights described before to obtain the forecasts arising from the expenditure and output components. 
Table 4 reports the RMSFEs comparing the GDP outturns to the GDP forecasts computed separately on the expenditure side and output side, respectively. We present two sets of results based on whether the weights of the forecast components to produce $\widehat{y_{t \mid t-h}^{\text {out }}}$ and $\widehat{y_{t \mid t-h}^{\text {exp }}}$ are estimated with increasing (upper panel) sample or rolling sample windows, as described before. The results are similar in both approaches. Importantly, the results in table 4 suggest that the output approach, in general, provides more accurate forecasts for GDP than the expenditure approach. Gains from using forecasts from the output approach instead of the expenditure are of $39 \%$ in RMSFE terms at the zero forecasting horizon, that is, for forecasts computed 30 days before the first release of quarterly GDP is due.

Table 4: Estimated weights for combining output and expenditure approach forecasts as in equation (1) and RMSFE for each approach.

\begin{tabular}{l|c|c|c} 
& $\mathbf{h = 2}$ & $\mathbf{h = 1}$ & $\mathbf{h = 0}$ \\
\hline Expenditure and Output App. weights estimated with increasing sample & \\
\hline $\boldsymbol{\lambda}$ & $1.121^{* * *}$ & $1.054^{* * *}$ & $0.888^{* * *}$ \\
& $(0.143)$ & $(0.113)$ & $(0.094)$ \\
RMSFE for expenditure approach & 0.506 & 0.465 & 0.407 \\
RMSFE for output approach & 0.319 & 0.264 & 0.249 \\
\hline RMSFE for combination (eq. 1) & 0.208 & 0.205 & 0.172 \\
RMSFE comb. gains to output approach : & $35 \%$ & $22 \%$ & $31 \%$ \\
\hline Expenditure and Output App. weights estimated with rolling sample \\
\hline $\boldsymbol{\lambda}$ & $1.012^{* * *}$ & $1.054^{* * *}$ & $0.874 * * *$ \\
& $(0.130)$ & $(0.112)$ & $(0.101)$ \\
RMSFE for expenditure approach & 0.515 & 0.501 & 0.426 \\
RMSFE for output approach & 0.310 & 0.268 & 0.252 \\
\hline RMSFE for combination (eq. 1) & 0.205 & 0.196 & 0.201 \\
RMSFE comb. gains to output approach : & $34 \%$ & $27 \%$ & $20 \%$ \\
\hline
\end{tabular}

Notes: The coefficients $\lambda$ are computed using equation (1) at different forecast horizons $h$ with forecasts for the expenditure and output approaches for 2008Q1 up to 2018Q3. Standard errors in parenthesis, ${ }^{* * *} p<0.01, * * p<0.05, * p<0.1$. The RMSEs are computed for forecasts for second part of the out-of-sample period (2013Q1-2018Q3).

Table 4 also presents the estimate of $\lambda$ in equation (1) at different forecast horizons over the period from 2008Q1 to 2018Q3. The estimate of $\lambda$ is smaller than 1 only at $h=0$, implying that the expenditure approach helps predict GDP only at the shortest horizon, while its contribution becomes negative at the other two longer horizons. 
Figure 1: Time Variation of $\lambda$ (weight coefficient on output-approach forecasts) for $h=2,1,0$ (in months).
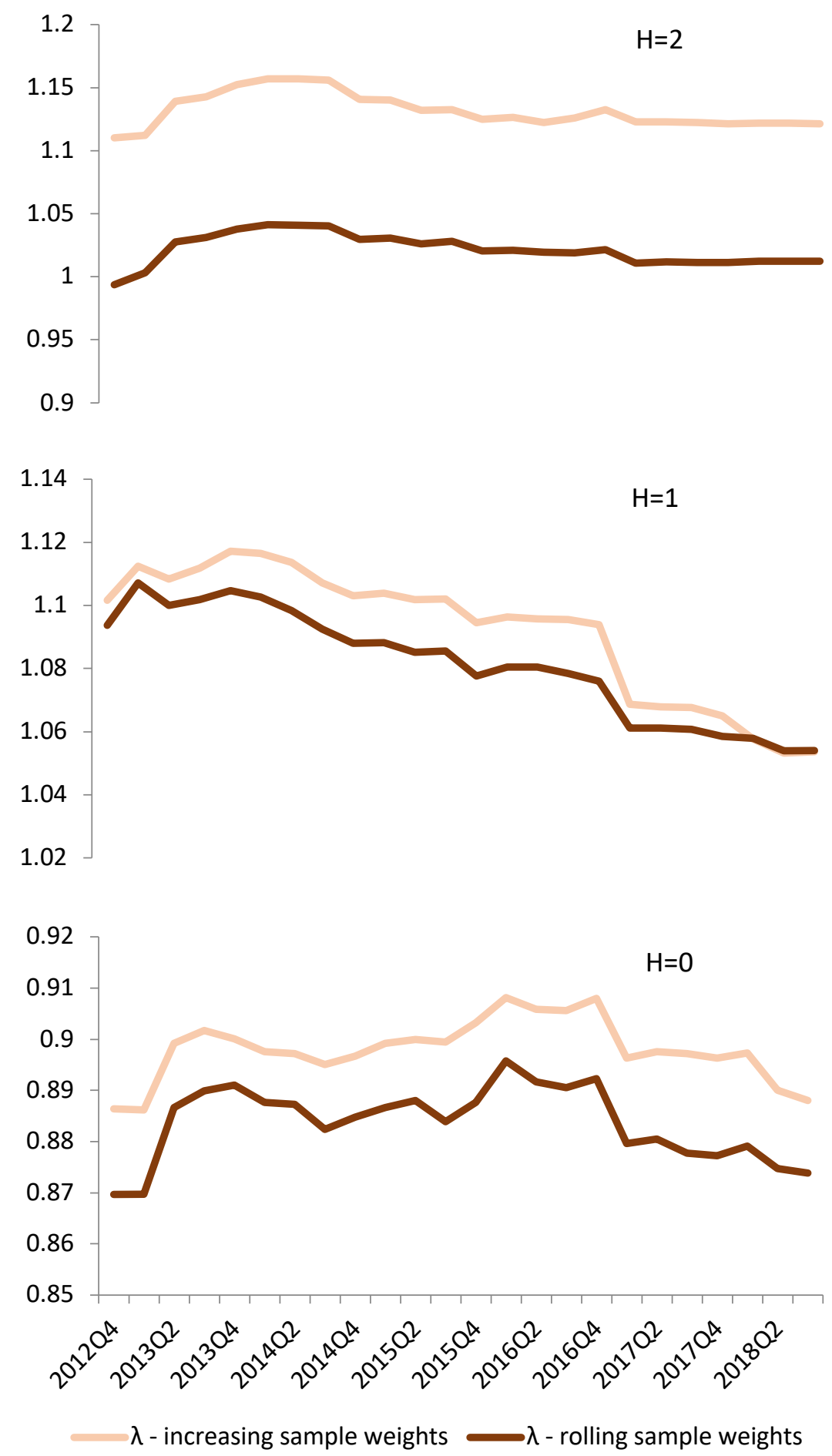

Note: Values smaller than 1 imply that a positive weight applies to forecasts from the expenditure approach. Values larger than 1 indicate that a negative weight applies to expenditure forecasts. 
Table 4 also includes the information of the RMSFE produced by the forecasting model described in equation (1), which aggregates the expenditure and the output approaches. In summary, although the output approach delivers smaller RMSFE than the expenditure approach over the 2013-18 period, a combination of both using weights based on forecasting performance since 2008 provides even more accurate forecasts over the period. We find accuracy improvements from combining both sets of GDP components even though the estimated weight on the expenditure forecasts are in general small and negative. These improvements are around $30 \%$ in terms of RMSFEs.

Figure 1 presents the evolution of the parameter $\lambda$ estimated in equation (1) over the period 2008Q1-2012Q4 using an increasing sample window up to 2018Q3. In general, it indicates little time variation, although the weight given to the expenditure approach (1- $\lambda$ ) increases a bit after 2017.

\section{2 - Evaluating the Coverage of Predictive Intervals}

As described in section 2.1, we use the weights in Figure 1 and estimates of the predicted variance to compute $50 \%, 75 \%$ and $90 \%$ predictive intervals for $h=0,1,2$ for the period between 2013Q1-2018Q3. Figure 2 illustrates the implied predicted intervals computed as described in section 2.2 and the outturns of the first quarterly GDP growth estimates. An inspection of Figure 2 suggests that the realisation -the ONS first quarterly GDP growth - is rarely outside the $50 \%$ predictive interval. Table 5 presents the empirical coverages for these predictive intervals. They suggest that predicted intervals are not well-calibrated, and that predicted variances are too large.

The sizeable predicted variances are due to the substantial forecasting errors recorded over the period employed to estimate weights - in particular during the Great Financial Crisis (2008Q2-2009Q2). In subsequent periods, the variability of the forecasting errors diminishes, specifically during the second part of the out-of-sample period (2013Q1-2018Q3). The regression employed to estimate weights and compute the predicted variance is not able to capture changes in conditional variance.

To model heteroscedasticity in the past forecasting errors, we run the regression in equation (1) with a generalized autoregressive conditional heteroskedasticity (GARCH) in the variance of the disturbances as described in section 2.1.1. The GARCH model estimates the 
conditional variance over the estimation sample. We use the conditional variance estimated for the last observation available in real-time as the predicted variance. Figure 3 presents the results for these real-time predicted intervals.

Figure 2: Nowcasting Predictive Intervals computed by weighting expenditure and output approach forecasts with weights estimated with increasing windows of data for $h=2,1,0$ (in months).

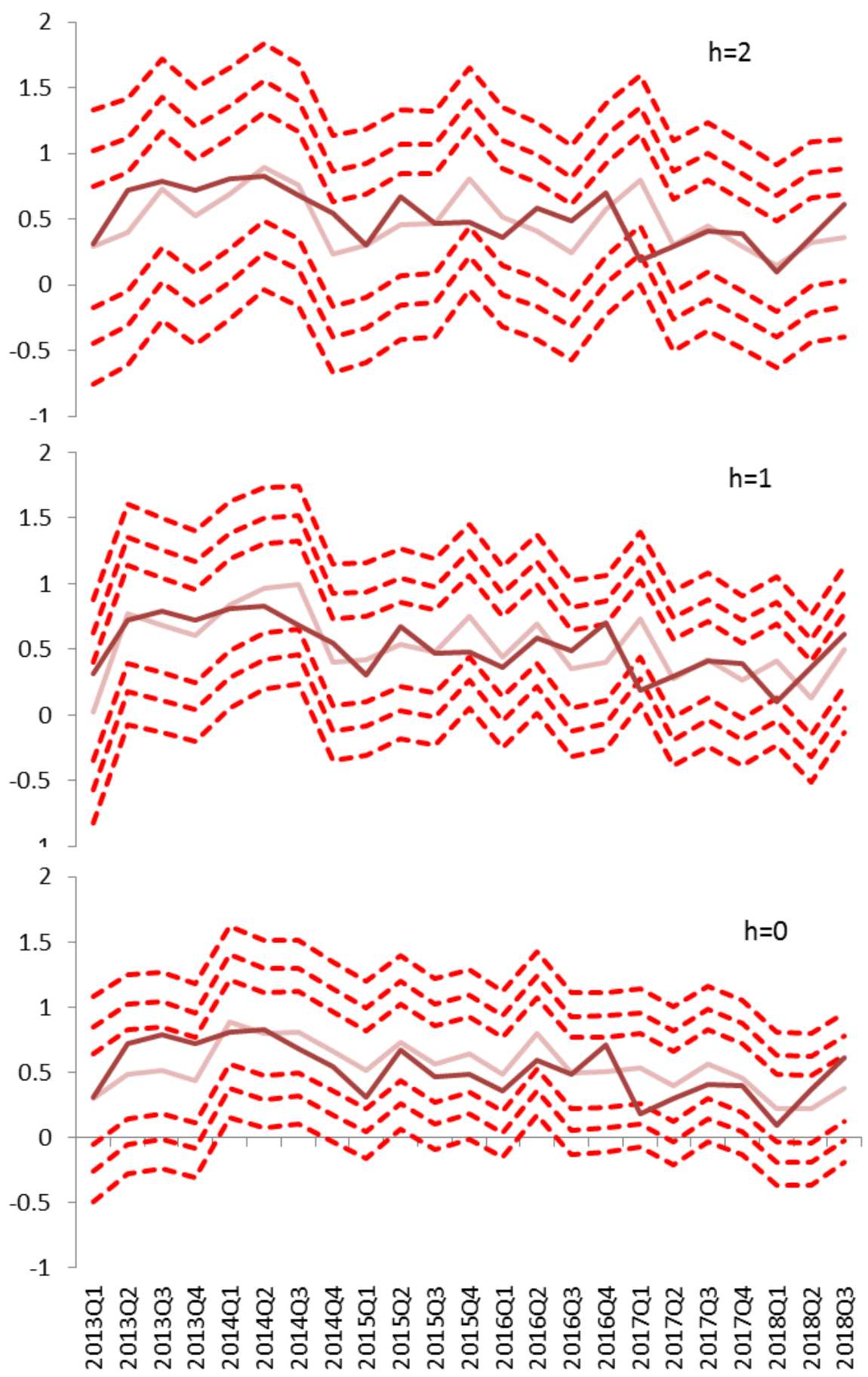

forecast y outturn y 
Figure 3: Nowcasting Predictive Intervals computed by weighting expenditure and output approach forecasts with weights estimated with increasing windows of data and with GARCH errors for $h=2,1,0$ (in months).

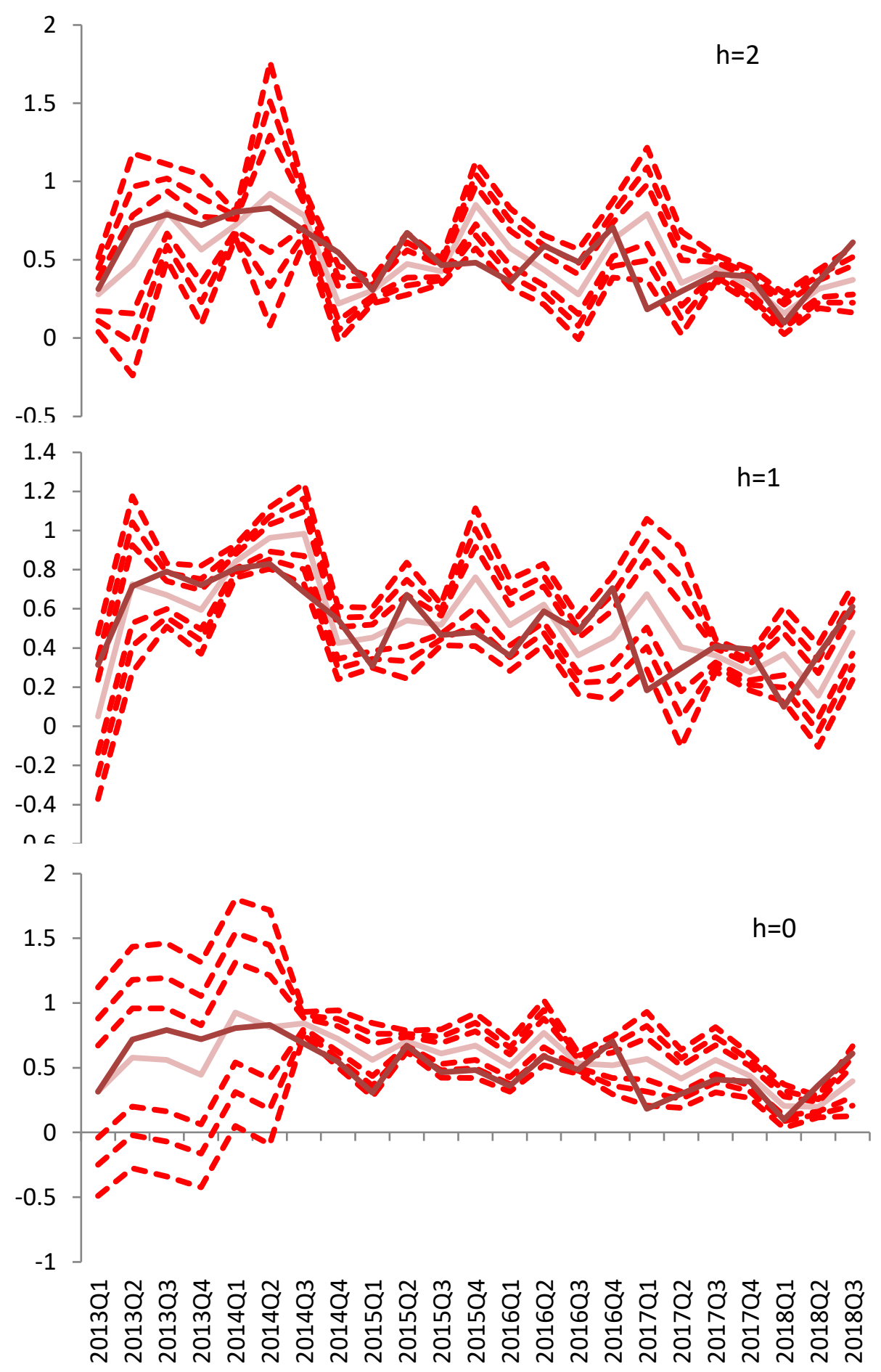

forecast y outturn y 
Comparing Figure 3 to Figure 2, we can see how by modelling changes in the conditional variance, we can obtain narrower intervals for all horizons. That is particularly the case after 2015. Table 6 presents the empirical coverage. For low nominal levels (50\% and $75 \%$ ), we find now some evidence of under-coverage. For the $90 \%$ level, we have coverages that are close to the nominal in particular for the two shortest horizons.

Table 5: Empirical Coverages with Combining Regression with constant variance

\begin{tabular}{l|lllllll}
\hline & \multicolumn{3}{|c|}{ Increasing Sample } & \multicolumn{3}{c}{ Rolling Sample } \\
\hline & $50 \%$ & $75 \%$ & $90 \%$ & $50 \%$ & $75 \%$ & $90 \%$ \\
\hline at $\mathrm{h}=2$ & 0.96 & 0.96 & 1.00 & 0.96 & 0.96 & 1.00 \\
at $\mathrm{h}=1$ & 0.87 & 0.96 & 1.00 & 0.96 & 0.96 & 1.00 \\
at $\mathrm{h}=0$ & 0.96 & 1.00 & 1.00 & 0.96 & 1.00 & 1.00 \\
\hline
\end{tabular}

Note: Each column heading indicates nominal coverage of the interval as entries are empirical coverages for predictions for the out-of-sample period between 201301 and $2018 Q 3$.

Table 6: Empirical Coverages for weighting regression with GARCH errors

\begin{tabular}{ll|lll}
\hline & \multicolumn{4}{c}{ Increasing Sample } \\
\cline { 2 - 5 } & & $50 \%$ & $75 \%$ & $90 \%$ \\
\hline at $\mathrm{h}=2$ & 0.43 & 0.65 & 0.74 \\
& at $\mathrm{h}=1$ & 0.17 & 0.56 & 0.83 \\
& at $\mathrm{h}=0$ & 0.35 & 0.52 & 0.87 \\
\hline
\end{tabular}

Note: See notes of Table 5.

In summary, the bottom-up nowcasting system can deliver well-calibrated predictive intervals for the ONS first release of quarterly GDP if we model the conditional heteroscedasticity in past forecasting errors before computing the predicted variance. The evidence of correct calibration is more convincing at the shortest horizon, that is, forecasts calculated 30 days before the ONS release of GDP.

\section{3 - A Comparison of the Nowcasting System with Professional Forecasts}

Table 7 shows the root mean square forecast error (RMSFE) for GDP point forecasts for the period 2013Q1-2018Q3. The first column displays results for our nowcasting system forecasts for the three horizons. In the remaining columns, we provide RMSFEs for a set of 
professional forecasters collected over the period 2013Q1-2018Q3. These include forecasts from the Bank of England Inflation Report (BofE), and NiGEM forecasts published in the National Institute Economic Review (NIESR). Both BofE and NIESR forecasts are nowcasts published during the second month of the reference quarter $(h=2)$.

Table 7: RMSFE of the Bottom-up Mixed-Frequency Nowcasting System versus Professional Forecasts

\begin{tabular}{c|c|c|c} 
& Nowcasting System & BofE, MPC & NIESR, NiGEM \\
\hline $\mathrm{h}=2$ & 0.20 & 0.20 & 0.28 \\
$\mathrm{~h}=1$ & 0.19 & & \\
$\mathrm{~h}=0$ & 0.18 & & \\
\hline
\end{tabular}

Note: The RMSFEs reported for the Nowcasting System are estimated using increasing windows and GARCH errors (see figure 3).

The results of the comparison of our nowcasting system to a set of professional forecasters suggest that our point forecasts are competitive. The nowcasting system improves RMSFEs in $10 \%$ in comparison with the most accurate professional forecast (Bank of England) as the system incorporates current quarter data when computing $h=0$. Worth noting that the nowcasting model presented in this paper is judgement-free, while NiGEM and Bank of England forecasts are not.

\section{5- Conclusions}

In this paper, we propose a bottom-up mixed-frequency approach to compute accurate nowcasts for the first ONS releases of UK quarterly growth. Our empirical results suggest that the nowcasting system can deliver well-calibrated short-term forecasts by combining forecasts of GDP components from both the expenditure and the output approaches. The substantial forecasting errors during the $2008 / 2009$ recession led to a temporary increase in forecasting uncertainty, which is modelled by assuming an autoregressive process in the conditional volatility of the forecasting errors. The modelling of this time variation on forecasting uncertainty has key role in obtaining predictive intervals with the adequate coverage. We also provide evidence that our nowcasts are competitive in comparison to the ones produced by professional forecasters such as the Bank of England and NIESR. 
One of the main advantages of our nowcasting system is that it is technically easy to implement in real-time by professional economists. All exercises in this paper were carried out using the software Eviews. We can then conclude that an accurate real-time nowcasting system for UK GDP could be used by economists that prefer to use a menu-based software as Eviews instead of code-based software such as Matlab. 


\section{6- References}

Anesti, N., Hayes, S., Moreira, A. and Tasker, J. (2017). Peering into the present: the Bank's approach to GDP nowcasting. Bank of England Quarterly Bulletin, Vol. Q2, pp. 122133.

Anesti, N., Galvao, A. and Aggripino-Miranda, S. (2018) Uncertain Kingdom: Nowcasting GDP and its revisions. Bank of England Staff Working Paper Series n. 764.

Bànbura, M., Giannone, D., Modugno, M. and Reichlin, L. (2013). Now-casting and the RealTime Data Flow. in Handbook of Economic Forecasting, volume 2A: Elsevier, pp. 195237.

Carriero, A., Galvão, A. and Kapetanios, G. (2019). A comprehensive evaluation of macroeconomic forecasting methods. International Journal of Forecasting. Forthcoming.

Clements, M. P. (2017). Assessing macro uncertainty in real-time when data are subject to revision. Journal of Business and Economic Statistics, 35:420-433.

Clements, M. P. and Galvão, A. B. (2008). Macroeconomic forecasting with mixed-frequency data: Forecasting output growth in the United States, Journal of Business and Economic Statistics. 26: 546-554.

Clements, M. P. and Galvão, A. B. (2013). Real-time Forecasting of Inflation and Output Growth with Autoregressive Models in the Presence of Data Revisions. Journal of Applied Econometrics. 28: 458-477

Clements, M. P. and Galvão, A. B. (2017). Data Revisions and Real-time Probabilistic Forecasting of Macroeconomic Variables.

Croushore, D. (2006) Forecasting with Real-Time Macroeconomic Data. in Handbook of Economic Forecasting I: Elsevier, pp. 96-982.

Foroni, C., Marcellino, M. and Schumacher, C. (2015). U-MIDAS: MIDAS regressions with unrestricted lag polynomial. Journal of the Royal Statistical Society, Series A. 178: 57-82

Galvao, A. and Michell, M. (2019) Measuring data uncertainty: an application using the Bank of England's 'Fan Charts' for Historical GDP Growth.

Higgins, P. (2014) GDPnow: A model GDP nowcasting. Federal Reserve of Atlanta Working Paper Series. 2014-7.

Mitchell, J. (2009) Where are we now? The UK recession and nowcasting GDP growth using statistical models. National Institute Economic Review. 209: 60-69.

Mitchell, J., Smith, R., Weale, M., Wright, S. and Salazar, E. (2005) An Indicator of Monthly GDP and an Early Estimate of Quarterly GDP Growth. The Economic Journal. 115: F108F129.

Pettenuzzo, D., Timmermann, A., Valkanov, R. (2016) A MIDAS Approach to Modeling First and Second Moment Dynamics. Journal of Econometrics. 193: 315-334 